Revue d'histoire de l'Amérique française

DEVUE D.HISTOIRE DE L'AMÉRIQUE FRANÇAISE

\title{
Les prétentions des religieuses de l'Hôpital-Général de Québec sur le palais épiscopal de Québec
}

\section{Micheline D’Allaire}

Volume 23, numéro 1, juin 1969

URI : https://id.erudit.org/iderudit/302853ar

DOI : https://doi.org/10.7202/302853ar

Aller au sommaire du numéro

Éditeur(s)

Institut d'histoire de l'Amérique française

ISSN

0035-2357 (imprimé)

1492-1383 (numérique)

Découvrir la revue

Citer cet article

D’Allaire, M. (1969). Les prétentions des religieuses de l'Hôpital-Général de Québec sur le palais épiscopal de Québec. Revue d'histoire de l'Amérique française, 23(1), 53-67. https://doi.org/10.7202/302853ar d'utilisation que vous pouvez consulter en ligne. 


\section{LES PRÉTENTIONS DES RELIGIEUSES DE L'HOPITAL-GÉNÉRAL DE QUÉBEC SUR LE PALAIS ÉPISCOPAL DE QUÉBEC*}

Le testament que l'intendant Dupuy, à titre d'exécuteur testamentaire, avait charge d'ouvrir après la mort de $\mathrm{Mgr}$ de Saint-Vallier, allait soulever de longues querelles au sujet du palais épiscopal de Québec. On pouvait s'attendre de la part de Mgr de Saint-Vallier à quelque chose de bien particulier quand on sait qu'il avait suggéré au Roi, quelques années avant sa mort survenue en 1727, de faire déclarer nuls les testaments qui ne laisseraient rien, soit à l'Hôpital-Général de Montréal, soit à l'Hôpital-Général de Québec où il vivait depuis 1713. Naturellement, le Ministre lui fit comprendre l'impossibilité de rendre un tel arrêt. ${ }^{1}$ D'ailleurs, avant même la lecture du testament, les religieuses savaient à quoi s'attendre car, avant de mourir, l'Évêque leur avait dit:

Je vous donne, mes chers enfants, dans mon testament le titre de légataires universelles. Vous n'y gagnez pas beaucoup, car vous n'ignorez pas que je suis très pauvre; ${ }^{2}$ aussi je ne vous charge que de très peu de choses. J'ai ménagé en France vingt-quatre-milles livres entre les mains de mon agent; vous les emploirez à ce qui sera le plus convenable pour l'avantage de cette maison. ${ }^{3}$

En effet, par son testament, daté du 25 mars 1725, SaintVallier - dont l'évêché se trouve réuni aux abbayes de Maubec et de Bénévent - lègue tous ses biens aux religieuses de l'HôpitalGénéral. ${ }^{4}$ L'exécution de ce testament va faire naître des difficultés tant en France qu'au Canada.

* Extrait d'une thèse de doctorat portant sur l'Hôpital-Général de Québec, présentée à l'Université d'Ottawa, le 2 octobre 1968.

1 Maurepas à Saint-Vallier, 30 mai 1724, AC, C11A, 47: 73-76.

2 Saint-Vallier avait été très généreux de son vivant en distribuant presque tout en aumônes; Taschereau, l'auteur de l'Histoire du Séminaire de Québec, dit qu'il aurait dépensé, seulement de son patrimoine, 200,000 livres (Taschereau, Histoire du Séminaire de Québec, 713).

3 Archives de l'Hôpital-Général de Québec, Annales de l'HôpitalGénéral de Québec, I: 341.

4 Testament de Saint-Vallier, 25 mars 1725, AC, C11A, 107: 332-341. 
A la nouvelle du décès et des dispositions testamentaires de l'Évêque de Québec, Saint-Senoch, conseiller du Roi, agent de la Communauté et exécuteur testamentaire de Saint-Vallier en France, entreprend de recueillir la succession au profit de la Communauté. Cette succession comprend des rentes sur l'Hôtelde-Ville et ailleurs, et le revenu de l'abbaye de Bénévent. Il s'empresse alors d'écrire, de Paris, à la Communauté:

... je vous avoue, Mesdames, que je comptais si peu sur la perte que nous venons de faire que j'avais déjà fait mes lettres et mes mémoires de recettes et dépenses et avais mis le tout dans une boîte avec quelques effets. Je prie M.D. de vous les faire tenir sûrement et en mains propres. Quand vous l'aurez reçue, vous ferez, Mesdames, tel usage que vous jugerez à propos de ce que vous trouverez dedans. Toute la grace que je vous demande, c'est de faire faire une copie du compte que je rendais à Monseigneur, et de la manière qu'il le faisait, et ensuite vous aurez la bonté de l'arrêtez en votre qualité de légataires universelles de feu Monseigneur. Vous le signerez, la communauté assemblée, n'y ayant personne qui soit en droit de le faire pour vous. Vous trouverez, Mesdames, que par ce compte j'étais redevable à Monseigneur de la somme de sept milles et tant de livres qui vous appartiendront au moyen du testament ci-dessus, après cependant que ce testament sera exécuté en ce qui concerne l'Ancienne France... Outre cette somme j'aurai à faire le recouvrement de l'année entière 1727 des rentes que Monseigneur avait sur l'Hôtel-de-Ville et dont il s'était réservé l'usufruit. D'ailleurs, je compte de toucher encore quelque chose de son abbaye de Bénévent, et ensuite quand j'aurai ramassé toutes ces sommes j'en fermerai un autre compte avec lequel je comprendrai le reliquat de celui de cette année que vous me ferez le plaisir de m'arrêter, et mettre en dépense les lettres de change qui me reste à payer de Monseigneur avec ce que je serai obligé de payer pour l'exécution de son testament. De cette façon vous connaîtrez parfaitement ce qui vous reviendra de votre legs universel. 5

Les religieuses se savent donc propriétaires d'une certaine somme de biens. Mais Saint-Senoch a pris garde de leur recommander la prudence en leur conseillant de ne point dévoiler les biens du défunt Évêque, étant donné que le coadjuteur, Mgr Dosquet, pourrait les faire saisir afin de procéder aux réparations de l'abbaye de Bénévent qui requièrent une somme considérable d'argent. C'est déjà plus qu'il n'en faut pour disposer

5 Annales de l'Hôpital-Général, I: 362-364. 
les religieuses de l'Hôpital-Général à soupçonner le Coadjuteur et à refuser toute perquisition chez elles.

Pour l'instant, de ce problème général de la succession, ne retenons que la question précise du palais épiscopal. ${ }^{6}$

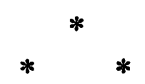

Nous savons qu'en 1688, Saint-Vallier a acheté, pour la somme de 15,000 louis, la maison qui devait lui servir de palais ainsi que le terrain où elle est construite. Lorsqu'il arrive à Québec, en 1697, il prend possession de son palais épiscopal. Cependant, après avoir perdu beaucoup d'argent à cause de la réduction de rentes sur l'Hôtel-de-Ville et avoir fait procéder à des constructions fort coûteuses à l'Hôpital-Général, il décide, en 1713, que ses revenus sont trop modiques pour continuer d'habiter au Palais. Il se retire alors à l'Hôpital-Général où il demeurera jusqu'à sa mort. Pendant ce temps, il loue le Palais au profit des pauvres. ${ }^{7}$ Nous savons, par exemple, que le médecin Sarrazin l'habite de 1720 à $1729 .^{8}$ Déjà, retenons alors que la location du Palais pouvait être une source de revenus.

Or, par son testament, Saint-Vallier lègue à ses successeurs évêques son palais épiscopal, à la condition toutefois de décharger sa succession des réparations à faire à l'abbaye de Bénévent. ${ }^{9}$ Voilà le cœur du problème: la possession du palais épiscopal est liée à la responsabilité de faire réparer cet abbaye.

Par son nouveau titre d'évêque du Canada, Mgr de Mornay se trouve pourvu de l'abbaye de Bénévent et, par conséquent, prend toutes les mesures pour le faire réparer aux frais de la succession de Saint-Vallier. Après avoir fait saisir les effets de la succession, il se fait autoriser par le Roi à procéder aux visites d'estimation des travaux. ${ }^{10}$ Saint-Senoch qui désire faire

${ }^{6}$ Sur la nature du palais épiscopal, nous référons le lecteur à $\mathrm{Mgr}$ H. Têtu, Histoire du palais épiscopal de Québec (Québec, Primeau et Kirouac, 1896), 19-26; ibid., appendice, 233-244.

7 Les Ursulines de Québec depuis leur établissement jusqu'à nos jours... (Québec, C. Darveau, 1863-1866), II : 163.

8 Pour connaître les noms de ceux qui habitent le Palais en l'absence de Saint-Vallier, voir Têtu, ibid., $44 \mathrm{~s}$.

9 Annales de l'Hôpital-Général, I: 368.

10 Ibid., 366. 
gagner quelques profits à l'Hôpital-Général se hâte alors de rencontrer Mornay pour tâcher d'en arriver à des arrangements profitables aux religieuses. La lettre que Saint-Senoch écrit à la Communauté de l'Hôpital-Général raconte assez bien les premiers mouvements de Mornay:

Je ne sais pas, Mesdames, si vous êtes informées du don que feu Monseigneur a fait à ses successeurs évêques du palais épiscopal qu'il a fait bâtir à Québec aux conditions (...) (qu'ils) ne pourront prétendre aucune chose ni rien demander de la succession de feu Monseigneur pour raison des réparations qui se trouveraient à faire lors de son décès dans les bâtiments et métairies des abbayes dont ils se trouveront titulaires, qui ont été unis au dit évêché. J'en ai l'acte en bonne forme, et je vous envoie un extrait, afin que vous puissiez vous en servir en temps et lieu. J'ai eu une très grande attention à faire connaître à Monseigneur de Mornay cette donation aussitôt que j'ai été informé de la mort de Monseigneur afin de le mettre en étât de l'accepter et de s'y conformer. J'ai même fait plus: dans l'instant que j'ai été instruit que le roi lui avait donné un coadjuteur et que ce serait lui qui passerait en Canada, j'ai jugé à propos de lui donner une copie de cette donation afin qu'il ne put l'ignorer. Cependant, nonobstant toutes ces précautions, et que cette donation paraisse très avantageuse pour Messieurs les Evêques de Québec, Monseigneur de Mornay n'a pas laissé de commencer par me faire signifier un acte le vingt-un juin, 1728, par lequel il s'oppose à ce que je me dessaississe, paye et vide mes mains des sommes que je puisse avoir et devoir à la succession et aux héritiers de défunt Monseigneur... Je l'allai trouver ensuite pour savoir à quel dessein il m'avait fait signer cet acte; il me dit que c'était pour arrêter les deniers que je pouvais avoir à feu Monseigneur pour les réparations qui étaient à faire à l'abbaye de Bénévent, et ce jusqu'à ce qu'il fut déterminé s'il accepterait ou refuserait l'acceptation ci-dessus. Depuis ce temps-là, j'ai fait, Mesdames, tout mon possible pour porter à prendre son partie, en lui faisant connaître que cela arrêterait tout, et entre autres choses le paiement de vos lettres de change; et enfin il n'a été pris ce parti qu'à la veille du départ de Monseigneur le Coadjuteur qui a été de refuser par eux cette donation. Il faut cependant rendre justice à Monseigneur de Mornay. Il a proposé à Monseigneur le Coadjuteur de l'accepter conjointement en lui offrant de se charger seul des réparations qui sont à faire à l'abbaye de Bénévent, ce qu'il a refusé de faire, disant qu'on l'avait assuré que le roi Louis XIV avait fourni des sommes pour acheter le terrain et faire le bâtiment de ce palais épiscopal; ce que voyant Monseigneur de Mornay il m'a fait faire ces jours-ci une nouvelle signification portant sommation de me 
transporter incessamment à l'abbaye de Bénévent pour être présent aux adjudications ou rabais des réparations qui y sont à faire, et même aux nouveaux procès-verbaux des réparations qui peuvent avoir été omises et de payer les adjudications des dites réparations au fur et à mesure de leur travail et jusqu'à la fin de paiement. Ensuite pour mettre toutes choses en règle et qu'il ne prétendit cause d'ignorance de la donation ci-dessus, je lui en ai fait donner copie avec sommation de s'y conformer. Il m'a répondu par une autre signification par laquelle il renonce purement et simplement à cette donation ...11

Après une phase d'hésitation, Mornay renonce donc à la donation du palais épiscopal. Saint-Senoch doit alors se rendre à Bénévent pour assister aux adjudications des travaux; tout ce qu'il peut obtenir est un rabais de 800 livres.

Mais ressentant le caractère un peu saugrenu de l'affaire, Mornay demande l'opinion du Ministre, en commençant par lui résumer les faits: d'une part, dit Mornay, Saint-Vallier a fait don du palais épiscopal à ses successeurs, palais qu'il a fait construire à ses propres frais, à la condition que sa succession ne soit pas tenue responsable des réparations; d'autre part, il a fait de l'Hôpital-Général son légataire universel. Or, accepter la donation, c'est se charger des réparations considérables de l'évêché et de celles de l'abbaye de Bénévent; la refuser, c'est remettre le Palais à la Communauté de l'Hôpital-Général et se décharger des réparations. Mornay ajoute que s'il ne pensait pas aux successeurs, il opterait pour la deuxième partie de l'alternative, mais qu'en songeant aux conséquences, il lui paraît raisonnable de ne pas laisser à l'Hôpital-Général une maison comme le palais épiscopal. ${ }^{12}$

$\mathrm{Au}$ moment où ont lieu les divers échanges de points de vue, les religieuses éprouvent de grandes difficultés dans leurs affaires temporelles. Selon l'Annaliste qui décrit la situation de 1730, la Communauté se retrouve avec près de 8,000 livres de lettres protestées; les lettres de change que les religieuses ont tirées, comme à l'ordinaire, sur Saint-Senoch et sur Saint-Vallier ne sont pas payées. En outre, les semestres de rentes et autres

11 Ibid.: 366-370.

12 Mornay à Maurepas, 24 janvier 1729, AAQ, Copies de lettres, v. II: 395. 
remboursements n'existent plus pour elles, car la saisie est posée sur tout ce qui concerne la succession du prélat. Pour faire face à leurs affaires, les religieuses ne trouvent que 1,830 livres de rentes. ${ }^{13}$ Par surcroît, Mornay veut faire réparer l'abbaye de Bénévent aux frais de la succession. La Communauté est vraiment dans une mauvaise posture matérielle, et c'est avec raison que le gouverneur propose alors au Ministre d'augmenter leurs gratifications.

Divers points de vue arrivent aux oreilles du Ministre. Beauharnois et Hocquart lui font entendre que la possession du Palais augmenterait sûrement les revenus de la Communauté; malheureusement, disent-ils, les religieuses n'ont en mains que le testament de Saint-Vallier "qui n'a point désigné la qualité des fonds qu'il leur laisse". Les papiers du défunt demeurent en la possession de Dupuy qui n'en a même pas fait l'inventaire. ${ }^{14}$ De son côté, le Coadjuteur prétend nulle la donation du palais épiscopal, car cette maison revient, de droit, aux successeurs de Saint-Vallier. Voilà précisément le point en litige qui, non seulement appelle différentes interprétations, mais qui soulève aussi des controverses plus ou moins subtiles.

Mornay lui-même, tâchant de se défendre de son mieux auprès du Ministre, raconte la mésentente qui existe entre lui et Dosquet sur la question du Palais.

Or, devant toutes ces difficultés exposées, le Ministre conseille à Mornay de renoncer à la donation onéreuse du palais épiscopal. Dosquet, au contraire, consent à l'accepter. Face au refus de Mornay, le Coadjuteur, en 1734, veut même lui intenter un procès. ${ }^{15}$ Nous savons, toutefois, que cette question n'est pas l'unique objet de dispute entre l'Évêque et le Coadjuteur.

De toutes façons, l'argumentation de Dosquet apparaît juste et fondée. Il y a une donation de faite en 1710, pense-t-il, par Saint-Vallier, à laquelle a renoncé Mornay, parce qu'il ignorait les titres à cette prétention. Ces titres sont dans le contrat de

${ }^{13}$ Annales de l'Hôpital-Général, II. La pagination du volume II est désordonnée.

14 Beauharnois et Hocquart à Maurepas, 10 novembre 1732, AC, C11A, 52 : 118-126.

15 Mornay à Maurepas, 20 mars 1734, AC, C11A, 62: 92-94. 
l'achat du terrain où l'on devait bâtir un palais épiscopal pour les évêques de Québec. De plus, le Roi a donné 10,000 livres pour la construction de ce Palais. Quant à Saint-Vallier, ajoute Dosquet, s'il a bâti ce Palais avec des aumônes ramassées, il ne considérait pas cette maison comme étant la sienne. ${ }^{16}$

Si l'argumentation de Dosquet est convaincante, les intentions de Sa Majesté sont, pour l'instant, assez nettes. La réponse du Ministre au Coadjuteur en fait foi:

Je dois cependant vous dire que par l'examen que Sa Ma. ${ }^{\text {té a }}$ fait de la prétention que ces Religieuses veulent exercer comme Legataires universelles de feu M. de S. ${ }^{t}$ Vallier, sur le Palais Episcopal, et de la représentation que vous luy avez faites a ce Sujet, Elle a reconnû que la donation faite en 1710 de cette maison par M. ${ }^{r}$ de S. ${ }^{t}$ Vallier a l'evêché de quebec et a Ses Successeurs Evesques, et là renonciation faite par M. de Mornay a cette donation le 9. juin 1729. sont deux titres également impuissants pour fonder cette prétention; qu'en effet, le bien que M. de S. ${ }^{t}$ Vallier paroissoit donner ne luy appartenoit pas; puisqu'il est dit dans le contrat d'acquisition qu'il avoit fait du terrain sur lequel la maison est Batie, que ce terrain étoit pour y Bastir un Palais Episcopal pour luy et Ses Successeurs Evesques a perpetuité, et, que Sa Majesté a donné $10 . \mathrm{m}$. pour cela, et que d'ailleurs M. de S. ${ }^{t}$ Vallier ne regardoit point ce terrain comme son bien qui luy fut propre, puisqu'il n'a jamais réclamé contre un arrest du Conseil Supérieur de Quebec qui l'avoit condamné a rendre le prix d'un emplacement qu'il avoit vendu Sur ce terrain, c'est par ces considérations que l'intention de Sa Ma. ${ }^{\text {té }}$ est que les Religieuses de l'hôpital général Se desistent de leur pretention a cet égard ... ${ }^{17}$

Le Roi pense donc que la donation que Saint-Vallier a faite du palais épiscopal ainsi que la renonciation de Mornay en 1729 sont des gestes insuffisants pour fonder les prétentions des religieuses. D'ailleurs, le palais épiscopal n'aurait jamais appartenu à Saint-Vallier.

Quelle part prend donc la communauté de l'Hôpital-Général dans ce conflit qui s'éternise? À vrai dire, c'est Saint-Senoch, l'exécuteur testamentaire en France, qui s'en occupe pour la plus grande part. C'est d'ailleurs le seul à pouvoir travailler à la cause avec compétence, ayant en mains les titres concernant la succession de Saint-Vallier. À Québec, les religieuses ne sont

16 Dosquet à Maurepas, 7 septembre 1731, AC, C11A, 56: 80-84.

17 Maurepas à Dosquet, $1^{\text {er }}$ avril 1732, AAQ, F. I-28. 
pas les seules personnes à se mêler de la question. En effet, c'est à Beauharnois et Hocquart que le Ministre s'adresse quand il s'agit de discuter de la succession. Il leur demande, par exemple, d'examiner, de concert avec le Coadjuteur, ce qui pourrait le mieux convenir à l'Hôpital-Général. ${ }^{18}$ Cependant, les religieuses de l'Hôpital-Général sont forcées de suivre ce long débat qui n'est nullement réglé après six ans. En effet, elles participent à toutes les tracasseries, étant donné qu'elles doivent faire passer plusieurs expéditions à Saint-Senoch, l'exécuteur testamentaire. Parmi ces expéditions, il en est une qui indique que les religieuses, aux moments importants, participent d'une façon active au conflit concernant le palais épiscopal de l'abbaye de Bénévent: c'est l'acte d'acceptation par la Communauté du legs de Saint-Vallier. Toutefois, les religieuses suspendent cette acceptation jusqu'à ce que le montant dû pour les réparations à l'abbaye de Bénévent soit définitivement fixé. ${ }^{19}$ Les religieuses, prudentes, attendent des éclaircissements pour opter définitivement; leur attitude est sage, puisque les réparations de l'abbaye sont une des charges les plus considérables de la succession.

Avant la fin de l'année 1733, le sort en est jeté: les religieuses n'ont même plus à délibérer car des lettres patentes du Roi viennent de porter don, au profit de Dosquet, des fruits et revenus du palais épiscopal avec la permission de disposer des bénéfices sujets à la régale. ${ }^{20}$ Peut-on comprendre que le Palais pouvait rapporter quelque profit? Nous sommes portée à le croire. Nous pensons aussi que c'est là un motif important qui fait prétendre aux religieuses de l'Hôpital-Général d'être en droit de le posséder. De toutes façons, les religieuses doivent s'incliner.

Ces patentes ne mettent pourtant pas fin aux nombreux ennuis concernant le Palais et les religieuses: Mornay continue d'affirmer que les religieuses de l'Hôpital-Général sont tenues, comme légataires universelles, de faire les réparations au Palais

18 Maurepas à Beauharnois et Hocquart, 20 mai 1732, AC, B 571: 311s.

19 Maurepas à Saint-Senoch, 6 janvier $1733, \mathrm{AC}, \mathrm{B} 58: 7 \mathrm{~s}$.

20 Lettre patente du Roi, 29 décembre 1733, AC, B 592: $366 \mathrm{~s}$. 
de l'évêché de Québec. Comme le souligne le Ministre lui-même, tout en exprimant la pensée de Saint-Senoch, Mornay n'est point facile en fait d'affaires. ${ }^{21}$ Cette remarque n'est pas surprenante pour peu que l'on connaisse Mornay. Il faut savoir, entre autres choses, que ce Capucin qui n'a été nommé évêque qu'à 50 ans, n'est jamais venu au Canada. Ce qui ne lui facilite évidemment pas la compréhension des problèmes. De leur côté, Beauharnois et Hocquart prennent position contre l'Evêque, disant au Ministre que les religieuses ne sont pas obligées de répondre aux prétentions de Mornay. Quant à l'attitude des religieuses, ajoutent-ils, "elles se tiendront sur la rescrue que $\mathrm{M}^{\mathrm{r}}$ de St-Senoch leur recommande. C'est en conséquence qu'elles remettent à l'année prochaine la main levée de la saisie que $\mathrm{M}^{\mathrm{r}}$ de Mornay a crû devoir faire sur elles des sommes estant entre les mains de $\mathrm{S}^{\mathrm{r}}$ $\mathrm{S}^{\text {t }}$ Senoch pour seureté de ces réparations". ${ }^{22}$

En réalité, les religieuses de l'Hôpital-Général n'ont point d'argent. C'est d'ailleurs ce que Beauharmois et Hocquart expliquent au Ministre ${ }^{23}$ tout en lui remémorant les faits. Rappelonsles brièvement. L'acquisition du terrain a été faite par SaintVallier en 1688, pour 15,000 livres: le Roi a payé 10,000 livres et l'Évêque, 5,000. Sur ce terrain, l'Évêque a fait ériger le palais épiscopal qui a coûté 40,000 écus, et qui a été légué à ses successeurs évêques le $1^{\text {er }}$ février 1710 , escomptant que ceux-ci déchargeraient ses héritiers des réparations à faire aux abbayes qui sont réunies à l'évêché. Mornay, son successeur, prend le parti de renoncer à la donation de 1710 et remet aux religieuses, légataires universelles de Saint-Vallier, les frais de réparations de ces abbayes qui coûtent plus de 10,000 livres. Les religieuses

21 Maurepas à Beauharnois et Hocquart, 17 mai 1734, AC B 611: 229s. C'est à plusieurs reprises que Mornay fait preuve de mauvais caractère. Nous savons qu'il est allé jusqu'à interdire les Jésuites en Louisiane: en 1732, il défendit au Supérieur des Jésuites et à tous les Jésuites sous ses ordres, d'exercer leurs fonctions ecclésiastiques. Nous constatons que ce Capucin peut causer beaucoup de problèmes. C'est d'ailleurs son comportement qui va amener Maurepas à le faire démissionner (C.E. O’Neill, Church and State in French Colonial Louisiana. Policy and Politics to 1732 (New Haven and London, Yale University Press, 1966), 230 ss.). 61: $23 \mathrm{~s}$

22 Beauharnois et Hocquart à Maurepas, 6 octobre 1734, AC, C11A,

${ }^{23}$ Beauharnois et Hocquart à Maurepas, 24 octobre 1737, AC, C11A, 107: $300-308$. 
pensent alors à revendiquer, comme compensation, le palais épiscopal à titre de bien compris dans la succession. Mais, en 1732, le Roi trouve leurs prétentions mal fondées. Se sentant fort, Mornay en profite pour demander leur exclusion du Palais. Pour gagner son point, Mornay invoque les raisons suivantes: le terrain du Palais a été acquis pour Saint-Vallier et pour ses successeurs; le Roi en a payé les deux-tiers; le terrain étant inaliénable, Saint-Vallier n'a pu céder de portion; ce dernier, enfin, a bâti sur un fonds dont il n'était qu'usufruitier.

Les religieuses de l'Hôpital-Général ne manqueront pas de se défendre, surtout à partir de 1737. Elles interviennent directement dans la lutte, par justice autant que par intérêt. Mère Duchesnay de l'Enfant-Jésus expose elle-même la situation au Ministre, en 1737: Mornay veut faire condamner les religieuses à payer les réparations, dit-elle, pourtant, il est bien stipulé, dans l'acte de donation de Saint-Vallier, que ses successeurs évêques ne pourront rien réclamer pour les réparations. Malgré tout, ajoute-t-elle, Dosquet qui est en possession du Palais depuis huit ans, a fait saisir des sommes provenant de la succession et qui auraient dû revenir à la Communauté. Mère Duchesnay demande alors, au nom de la justice, un remboursement des 10,000 livres pour les réparations faites aux abbayes, de même qu'une gratification. ${ }^{24} \mathrm{Ce}$ placet a sans doute sensibilisé Sa Majesté, puisqu'elle donne au moins, aux religieuses, la liberté de faire valoir leurs prétentions sur la maison épiscopale. ${ }^{25}$ Jusqu'en 1739, le Roi demeure résolu de défendre les religieuses contre les injustices, car on lit, dans le mémoire annuel:

Si par l'Examen qui sera fait des pretentions de cet hopital sur la maison Episcopale, il paroit qu'Elles peuvent etre fondées, S.M. souhaite qu'il ne soit intenté a leur nom aucune action sur cela sans que les S. ${ }^{\text {rs }}$ de Beauharnois et hocquart en ayent auparavant rendu compte, et qu'Elle leur ayt fait savoir ses intentions a ce sujet. ${ }^{26}$

Sans doute un peu ébranlé par l'allure décidée du Roi, peut-être un peu lassé des choses qui traînent, l'Évêque de

24 Placet de Mère Duchesnay, supérieure de l'Hôpital-Général, octobre 1737, AC, C11A, 107: 309-316. 179-182

25 Maurepas à Beauharnois et Hocquart, 15 mai 1738, AC, B 66:

26 Mémoire du Roi, $1^{\text {er }}$ mai 1739, AC, B 681 : 155-185. 
Québec, en 1739, fait la proposition suivante au Ministre: le Roi achèterait le Palais à l'Hôpital-Général pour une somme modique afin de pouvoir, par la suite, en faire l'usage qu'il lui plaira; "par ce moyen, ajoute-t-il, on coupera court aux proces qui seroient tres couteux aux successeurs de M. de S. ${ }^{t}$ Vallier et a l'hopital General". ${ }^{27}$

Sans désapprouver l'idée, le Ministre lui rend cette réponse circonspecte:

J'ajouteray deux observations par rapport a cet arrangement: L'une, que les Relig.es de l'hop. ${ }^{1}$ general ont $f .^{e}$ des reparations a l'abbaie de Benevent pour une so.e de 9252.\#11. ${ }^{\mathrm{s}}$ et qu'en demandant aujourd'huy d'estre maintenües dans la propriété et possession du Palais Episcopal, Elles offrent d'en faire l'abandon, pourvu qu'on leur rembourse cette so.e avec les interests a compter du jour du payement qui en a est. fait; Et l'autre que les deux tiers du terrain sur lequel cette maison a esté batie ont est. payés des deniers du Roy, et que par cette raison M. de S. ${ }^{\mathrm{t}}$ Vallier qui n'estait que l'usufruitier de ces deux tiers, n'avait pû en disposer. ${ }^{28}$

On voit que le Roi ne se montre pas enthousiaste à payer la somme exigée par les religieuses.

Le Ministre demande alors à Beauharnois et à Hocquart un mémoire concernant l'état et la valeur de la maison épiscopale avec ses dépendances, ainsi que de la somme des réparations à faire, avant d'arriver à un arrangement avec la communauté de l'Hôpital-Général. ${ }^{29}$ Tout ne va pas sans encombre. Après avoir recueilli ses informations, Dosquet s'empresse d'affirmer au Ministre que le Palais n'appartient pas à l'Hôpital-Général, mais bien aux évêques, étant donné que le clergé a accordé une pension de 2,000 livres pour bâtir ce Palais de Québec; il faut donc l'acheter, non plus de l'Hôpital-Général, mais bien des évêques. D'ailleurs, ajoute-t-il, ce serait avantageux pour les religieuses d'être déchargées des réparations auxquelles elles sont tenues comme légataires universelles de Saint-Vallier. Puis Dosquet précise que le coût du Palais est de 40,000 écus et la valeur actuelle du terrain est de 50,000 livres; en l'achetant, le Roi pourrait donner aux évêques une pension de 2,000 livres avec concession d'une

27 Dosquet à Maurepas, 11 juin 1739, AC, C11A, 72: 104-106.

28 Maurepas à Dosquet, 16 juin 1739, AC, B 69: $78 \mathrm{~s}$.

29 Maurepas à Beauharnois et Hocquart, 16 juin 1739, AC, B 681 : 54. 
terre, en les obligeant d'utiliser cette somme pour mettre la terre en valeur jusqu'à ce qu'elle rende 1,000 écus; après quoi le Roi retirerait sa pension. ${ }^{30}$

Il faut souligner que la situation faite à Dosquet au moment où il s'exprime, est plutôt pénible: il n'a pas encore récupéré, en 1739, les 12,000 livres qu'il a avancées 10 ans plus tôt pour payer les ouvriers; ceux-ci réclamaient alors leurs salaires dus pour des travaux faits au Palais. ${ }^{31}$

Quand on songe que l'incertitude au sujet du palais épiscopal règne encore en 1740 , on peut comprendre l'humeur peu commode de Dosquet qui, de nature, aimait l'ordre. En effet, le Palais peut encore, en 1740, appartenir, soit au Roi, soit aux évêques de Québec, soit aux religieuses de l'Hôpital-Général. A qui finirat-il par être donné? Chose certaine, les religieuses peuvent prétendre à bon droit à sa possession, car Mornay les a forcées à faire les réparations des abbayes et cela, contre la condition du don fait aux évêques de Québec.

Enfin, avec un ton qui, après 13 ans de luttes, nous semble un peu humoristique, le Roi décide de trancher la question en mars 1741, sous les pressions de Mgr de Pontbriand, afin, dit-il, d' "éviter les discussions qui pourroient s'elever au sujet des reparations a faire a la maison Episcopalle". Un premier arrêt est rendu le 12 mai 1741 par lequel Sa Majesté ordonne à Chaussegros de Léry, ingénieur au Canada, et à des experts de dresser un procès-verbal des réparations à faire au Palais. ${ }^{32}$ Puis, pour terminer les contestations avec les évêques, Sa Majesté décide d'indemniser l'Hôpital-Général. Un second arrêt ordonne ensuite que l'on procède aux réparations avec les deniers du Roi. Ainsi, l'Hôpital-Général et Dosquet avec ses successeurs deviennent quittes et déchargés du coût de toutes les réparations dont Sa Majesté leur fait don. ${ }^{33}$

30 Dosquet à Maurepas, 10 juillet 1739, AC, C11A, 72: 107-109.

31 L'épisode est raconté par A. Gosselin, L'Eglise du Canada depuis Monseigneur de Laval jusqu'à la Conquête (Québec, Laflamme et Proulx, 1911-1914), II : 62-64.

32 Arrêt du Conseil d'Etat, 12 mai 1741, AC, B 72: 258-260. Selon le procès-verbal, la somme des réparations monterait à 10,080 livres. $13: 65$.

33 Arrêt du Conseil d'Etat, 20 avril 1742, AC, Moreau Saint-Méry, 
Peut-on le croire? Le Roi hésite encore à rejeter les prétentions des religieuses, et attend d'être informé des bénéfices qu'a pu recevoir l'Hôpital. ${ }^{34}$ Mais il faut voir, dans tout cela, une question d'affaires et non de générosité: la Cour persiste à vouloir connaître ces bénéfices, parce que, précisément, si les religieuses en ont réalisés, elles devraient voir aux réparations de l'évêché que le Roi doit maintenant payer. ${ }^{35}$

En 1743, les prétentions de l'Hôpital-Général n'ayant pas été trouvées fondées, Sa Majesté dédommage l'Hôpital-Général et réunit définitivement le Palais à son domaine; en même temps, il en assure la possession et la propriété aux évêques de Québec.36

Cette longue histoire qui a duré quinze ans, concernant la possession du palais épiscopal de Québec est, avant tout, un conflit juridique; et c'est ainsi que le voient les religieuses de l'Hôpital-Général quand elles prétendent pouvoir posséder le Palais. Elles se sont prévalues de la clause de 1710 : elles étaient en droit de le faire puisque, d'une part, elles avaient fait procéder aux réparations de l'abbaye de Bénévent uni à l'évêché de Québec et que, d'autre part, Mornay avait renoncé à la propriété du Palais. Par conséquent, leur attitude est logique et leurs prétentions, justes. Mais, au-delà de ces faits, se pose une question extrêmement importante: la donation faite par Saint-Vallier n'était-elle pas illusoire? C'est sur cette question qu'ont reposé les différentes argumentations.

La part prise par les religieuses dans cette lutte nous révèle quelques traits de leur mentalité. D'abord, elle savent se défendre. Si au début de la lutte - comme nous l'avons vu - elles suivent le conflit, sans intervenir autrement qu'en faisant des expéditions de titres et d'autres documents, vers la fin, elles montent sur la scène du drame pour s'affirmer devant Dosquet comme devant Mornay. Cette attitude est d'autant plus courageuse que les religieuses sont loin de pouvoir aborder amicalement l'un et l'autre évêque: le premier, Mornay, n'est pas facile

\footnotetext{
34 Maurepas à Pontbriand, 27 avril 1742, AC, B 742: 353-358. 375-377.

35 Maurepas à Beauharnois et Hocquart, 27 avril 1742 , AC, B $74^{2}$ :

36 Arrêt du Conseil d'Etat, 30 mai 1743, AC, B 76'1 : 402-410.
} 
en affaires; le second, Dosquet, ne nourrit aucune espèce de sympathie à l'égard de la communauté de l'Hôpital-Général. Malgré tout, les religieuses, au nom de la justice et du droit, se lanceront dans la lutte jusqu'à vouloir intenter un procès à l'Évêque. Voilà une belle lutte juridique qui n'est pas seule en son genre, mais qui illustre plutôt l'époque où l'on aime à se battre au nom du principe, de la règle, du droit. Et l'on a beau jeu en Nouvelle-France de compliquer et d'éterniser les questions, étant donné l'éloignement du Roi qui n'arrive à percevoir les problèmes qu'avec beaucoup de lenteur, comme nous venons de le constater par cette étude.

La question du palais épiscopal n'est pourtant pas qu'une lutte juridique, car les religieuses avaient un intérêt matériel à l'emporter; en effet, elles auraient pu profiter des revenus des locataires. De plus, n'y aurait-il pas eu quelques bénéfices à retirer des abbayes? C'est d'ailleurs cette question d'intérêt qui nous permet davantage d'apprécier l'attitude de la Communauté tout au long de la lutte. En effet, elle s'interroge sans cesse sur les avantages de la succession: est-elle plus onéreuse que profitable?

Tout compte fait, cette prétention des religieuses de l'Hôpital-Général dans les bénéfices du palais épiscopal n'est pas aussi singulière qu'on est porté à le croire, à priori: les Ursulines établies aux Antilles ont, elles aussi, eu des prétentions semblables, dans la deuxième partie du XVII ${ }^{e}$ siècle. En effet, lorsque Bégon - président du Présidial de Blois, nommé intendant des îles d'Amérique en 1682 - arrive aux Antilles, il est d'abord obligé de lutter contre les Ursulines qui avaient acquis le palais de l'Intendance des mains de son prédécesseur. ${ }^{37}$

La question un peu saugrenue du palais épiscopal nous révèle que les Hospitalières de l'Hôpital-Général ont le sens des affaires et aussi une mentalité légaliste assez prononcée; en un mot, ce sont des femmes réalistes. Nous savons par ailleurs, qu'elles sont vraiment en contact avec le monde environnant, qu'elles connaissent les principaux problèmes politiques et éco-

37 F. Mauro, L'expansion européenne 1600-1870 (PUF, Nouvelle Clio $27,1964), 192$. 
nomiques de la colonie, qu'elles suivent les événements de très près et même qu'elles prennent part aux luttes les plus complexes de la société; et cela avec compétence et assurance. Nous constatons que les religieuses "cloîtrées" de l'Hôpital-Général sont loin de vivre isolées à tout jamais dans leur monastère.

Université d'Ottawa

\section{MICHELINE D'ALLAIRE}

\section{LIVRES REÇUS :}

Bibliothèque nationale du Québec, Index alphabétique du catalogue systématique selon la classification Library of Congress. Ministère des Affaires cultures, 1969. La Bibliothèque Nationale, en publiant cet index et en le diffusant largement, espère fournir aux bibliothèques du Québec un nouvel instrument de travail qui facilitera l'utilisation des tables de classification "Library of Congress". Cet ouvrage constitue, en définitive, un index cumulatif français des tables "Library of Congress".

Pour construire l'Eglise nouvelle - Normes d'application du Concile Vatican II et documents complémentaires. Texte original latin et traduction française. Tome I: 1963-1966. Fides, 245, boul. Dorchester, Montréal. 414 p. $\$ 8.95$.

Jean-Claude Drolet, L'Ordre des Chevaliers de Colomb - Origine, nature, structure, initiation, implantation dans la province de Québec. Centre d'études et de recherches historiques du Saguenay, Séminaire de Chicoutimi, 1968. 253 p. "En écrivant ce livre, l'auteur ne veut céder à aucune intention polémique... Notre étude veut être une histoire générale de l'Ordre".

Philippe Bergeron, L'action humaine dans l'œuvre de Teilhard de Chardin. Coll. "Philosophie et problèmes contemporains". 324 p. Fides, 245 est, boul. Dorchester, Montréal 129. $\$ 5.00$.

Edouard Parent, o.f.m., Ephrem Longpré, un mystique franciscain de notre temps - Journal spirituel et Lettres présentés par Edouard Parent, o.f.m. Bibliothèque de spiritualité, Les Editions Beauchesne, Rue de Rennes, 117, Paris, VIe, 1969. 42F. Le Père Ephrem Longpré, médiéviste et théologien, né aux Etats-Unis en 1890 de parents canadiens-français - dont il garda la nationalité canadienne - mourut à Paris en 1965 après avoir vécu en France depuis 1939. 